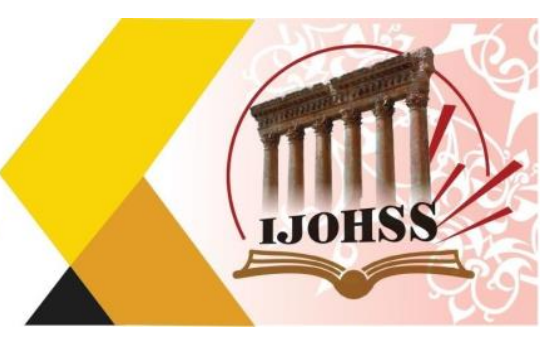

\title{
اللغة العربية، تحديات الواقع وأسرار البقاء
}

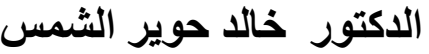 \\ جامعة ذي قار - كلية الآداب \\ العراق
}

الملخص

المنطلق في هذه الورقة، بيان قيمة التحديات التي تو اجه العربية، وبيان صلادتها في الوقت نفسه، لتكون

$$
\text { عصية على الاستسلام. }
$$

فيشتغل هذا البحث على أربعة مفاصل الأول، قيمة اللغة في فكر علماء العربية القدماء، والمحثثين، ومنهم ابن

فارس، وابن جني، و عباس محمود العقاد، ومجد الباكير. و المفصل الثاني التحديات التي تواجه العربية، وهي: العامية، ومواقع التواصل الاجتماعي، والازدواج اللغوي، والعولمة، ووسائل الإعلام، وشعبوية التعليم ،

$$
\text { و الانحر اف الديني الحاصل في المجتمع. }
$$

و المفصل الثالث أسر ار قوة العربية، ومواجتها للتحديات، وهي: رصانة الثعر التي كتب بها، وجود القرآن

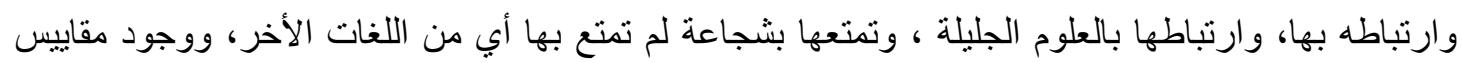

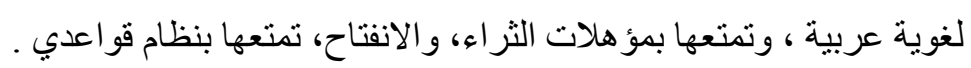
و المفصل الر ابع و هو الخاتمة وتبيان علاقة اللغة بالهوية، فمتى ما فرطت العربية ببعض أسرارها، نمانه تم التفريط

$$
\text { بهوية الفرد و المجتمع. }
$$

الكلمات المفتاحية: اللغة العربية. 


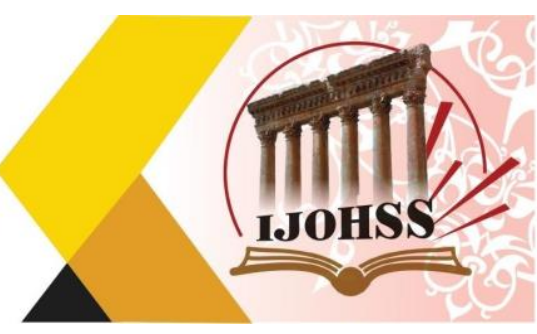

\title{
The Arabic Language, challenges Reality and Secrets of Survival
}

\author{
Dr. Khaled Huwair Al-Shams \\ Dhi Qar University - College of Arts \\ Iraq
}

\begin{abstract}
The starting point of this paper is to demonstrate the value of the challenges facing the Arab world, and to demonstrate their strength at the same time, so that they are resistant to surrender.

This research works on four chapters, the first is the value of language in the thinking of ancient Arab scholars and modernists, including Ibn Faris, Ibn Jani, Abbas Mahmoud al-Akkad, and Majd al-Bakir, the second detail is the challenges facing Arabic, which are: colloquial, social media, double language, globalization, the media, education populism, and the religious deviation in society .

The third detail is the secrets of the strength of Arabic and its confrontation with challenges, which are: the sobriety of the poetry with which it was written, the existence of the Qur'an and its association with it, its connection with the venerable sciences, its courage that none of the other languages enjoyed, the presence of Arabic linguistic standards, its enjoyment of the qualifications of wealth and openness, its enjoyment of a grammatical system .

The fourth joint, which is the conclusion and the clarification of the relationship of language to identity, so when Arabic abandoned some of its secrets, the identity of the individual and society was forfeited .
\end{abstract}

Keywords: Arabic Language. 


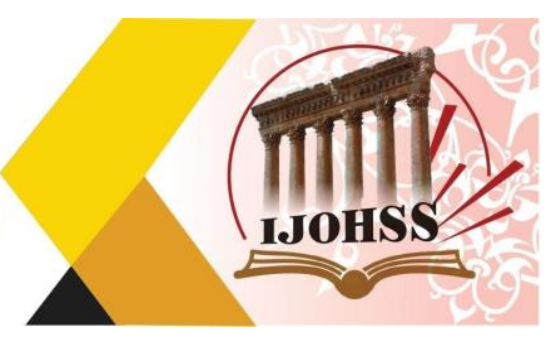

المقدمة

الحمد لله ، و الصلاة و السلام على رسول اله، و على أهل الدين كلهم...

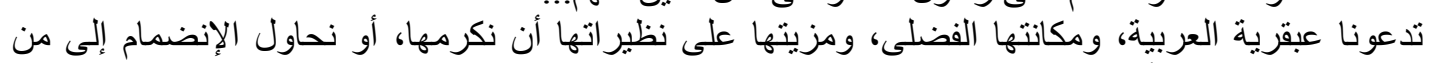

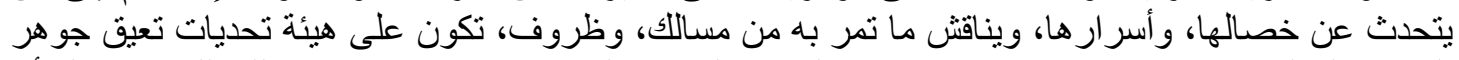

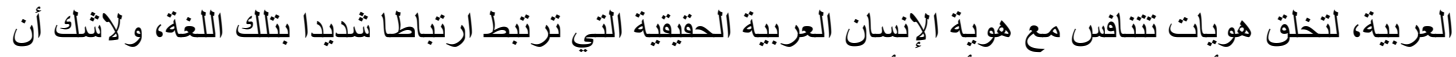

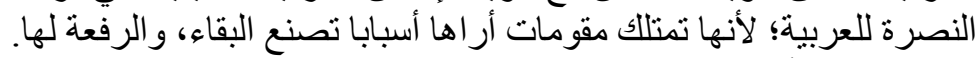

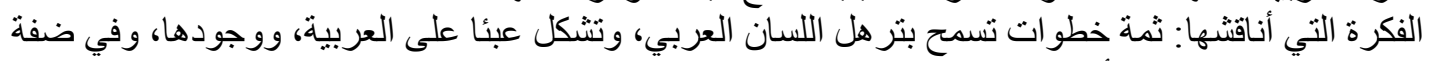

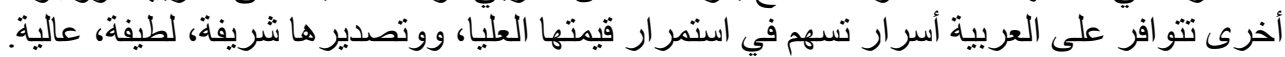

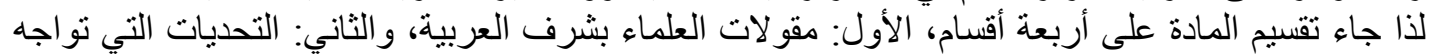

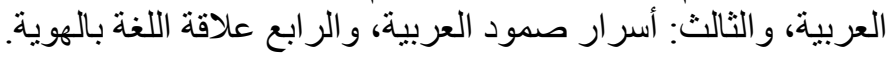

\section{القسم الأول : قيمة العربية في منظور العلماء}

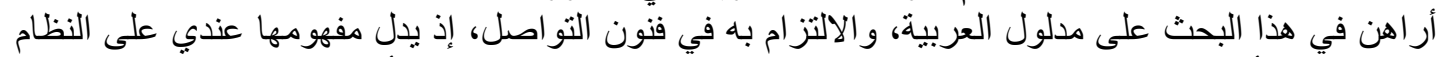

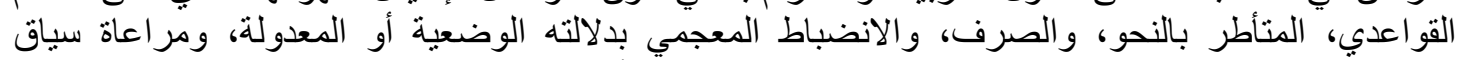

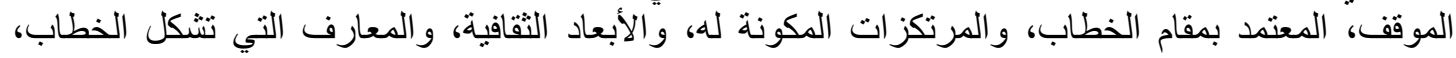

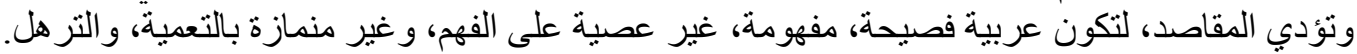

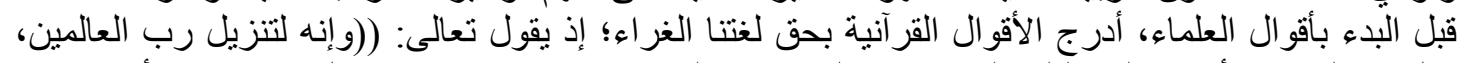

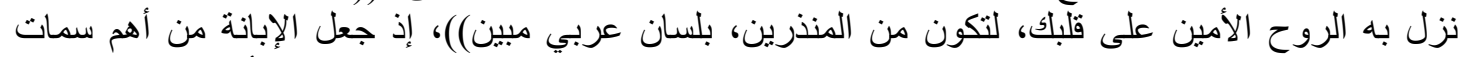

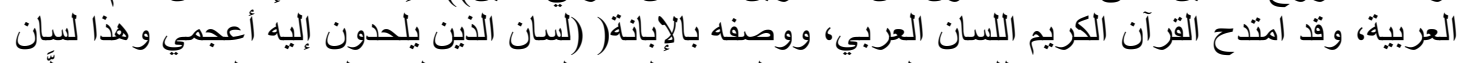

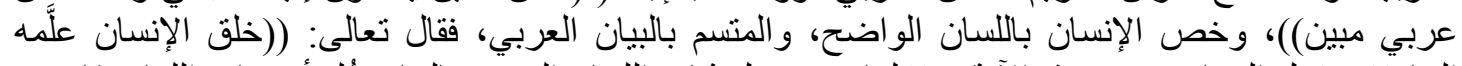

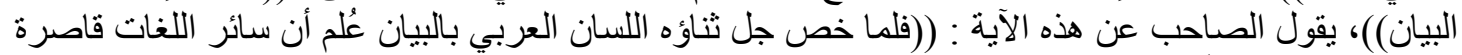

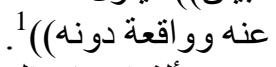

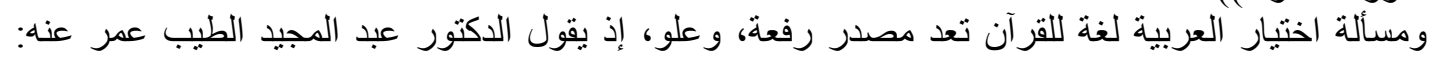

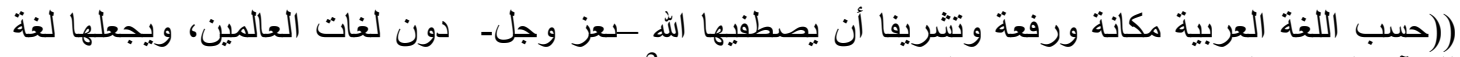

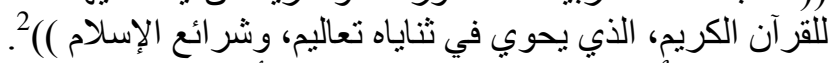

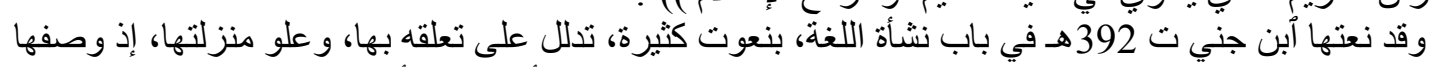

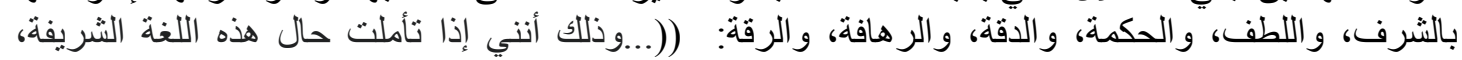

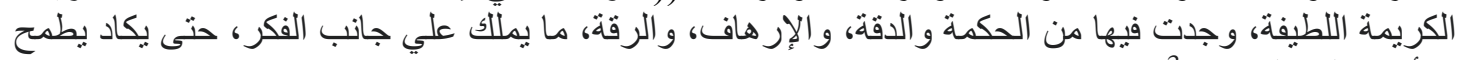

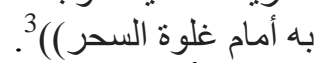

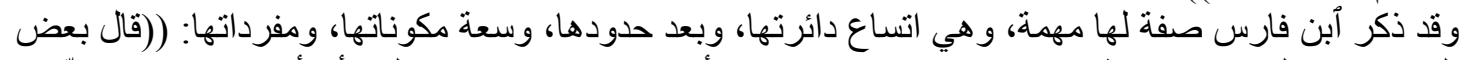

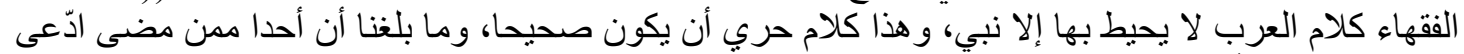
حفظ اللغة كلها)(أ).

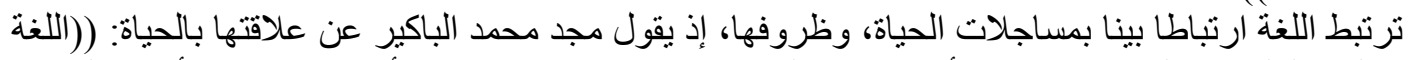

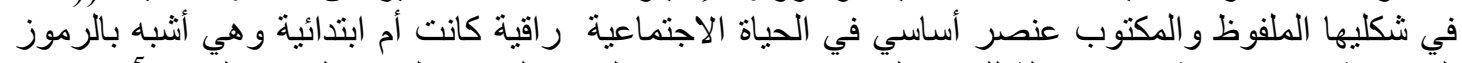

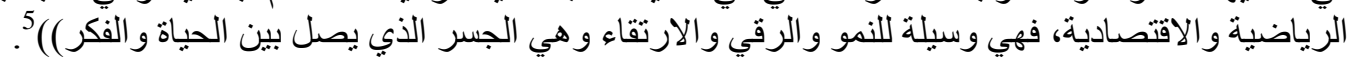

1 الصاحبي في فقه اللغة وسنن العرب في كلامها، أبن فارس: 16 فئل 16.

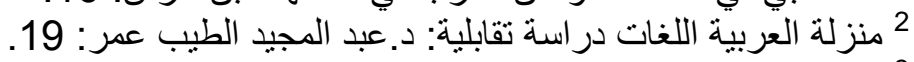

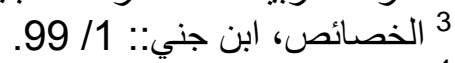

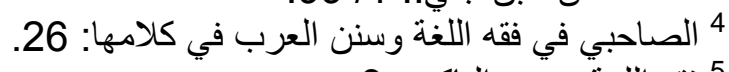
5 


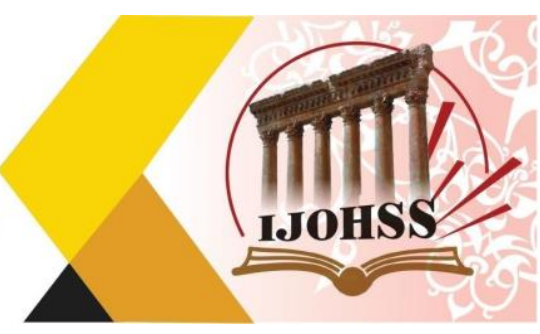

ويقول العبقري العقاد عباس محمود عنها بانها لغة شاعرة (وليس في اللغات التي نعرفها، أو نعرف شينًا كافيًا

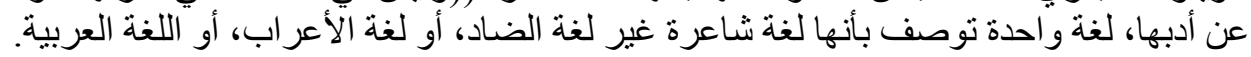

وتقدم أننا لا نعني باللغة الثـاعرة ما يوصف أحيانًا باللغة الشعرية، فإن الكلمة قد تكون شعرية صالحة للنظم

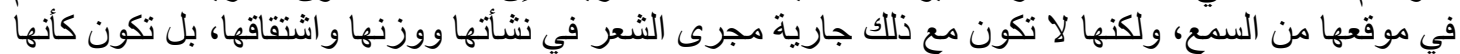
الطعام الذي يصلح لتركيب البنية، ولكنه هو في ذاته ليس بالبنية الحية، وليس باللحم و الدم الذي ينركب الذب منه أجسام

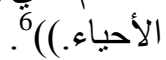

وقد يمتدح الدكتور عبد المجيد الطيب عمر النظام الصوت في العربية ، فيقول في مقدمة كتابه (منزلة العربية

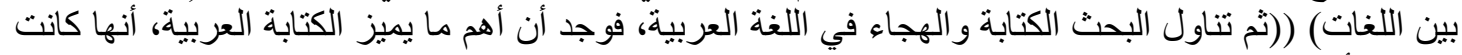

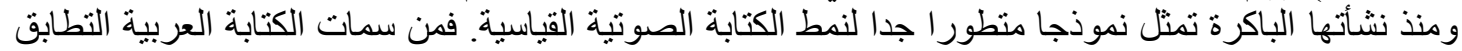

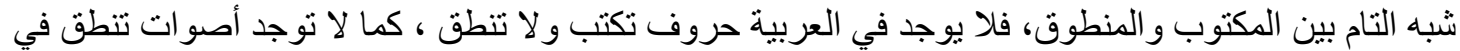

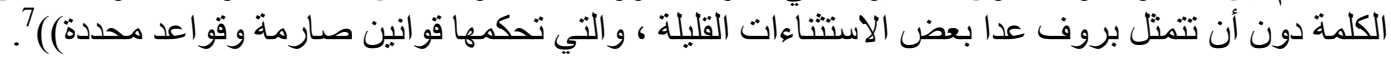

وقد جعلها ناصر الدين الأسد وطيدة الصلة بالهوية والثقافة: (وتتبلى أهيّة اللّّة العربيّة في العلاقة الوطيدة

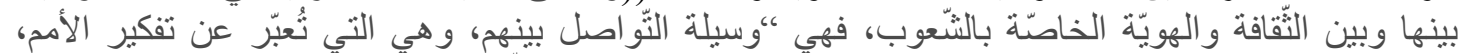

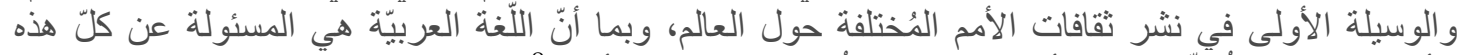

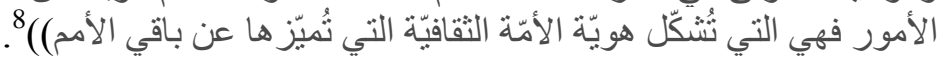

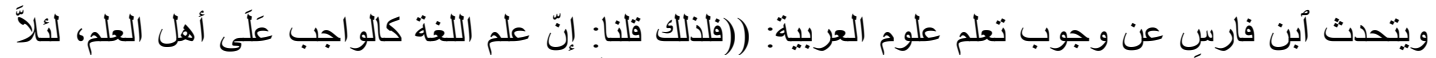

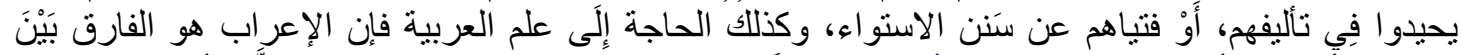

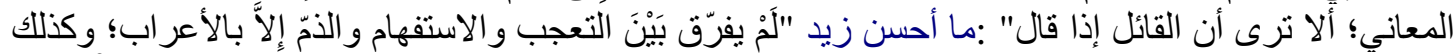

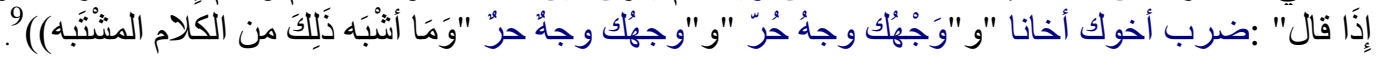
وقال اللساني فان دايك عنها: ((وقال" فان ديك) "الأمريكي" :((العربيةُ أكثر لغات الأرض امتيازًا، وهذا

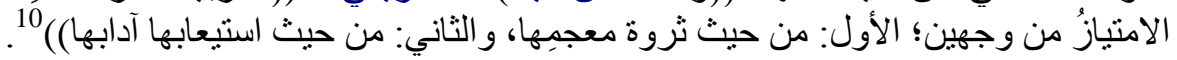

\section{القسم الثاني : التحديات التي واجهت العربية}

و أقصد بالتحديات الرهانات التي تمر بها العربية، وتشكل خطر ا على حضور ها، وطريقة أدائها، من جهة

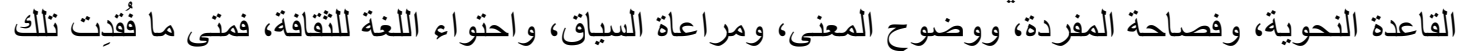

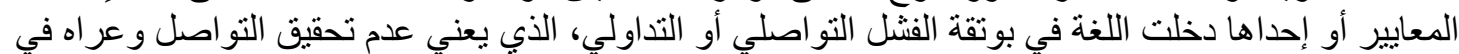

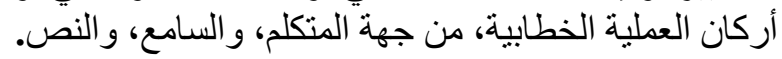

1- العامية: لم تكن مشكلة العدول نحو العامية مشكلة وليدة اليوم، بل هي متجذرة في الفضاء الاستعمالي

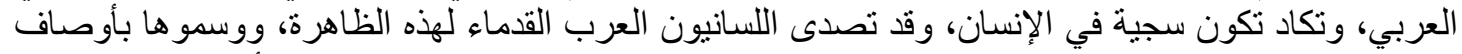

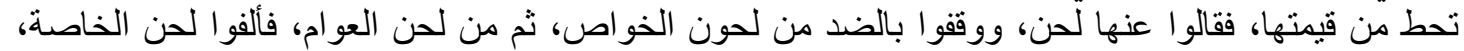

6 - اللغة الثاعرة، العقاد: 12.

7 منزلة اللغة العربية بين اللغادة اللغات دراسة تقابلية: 8.

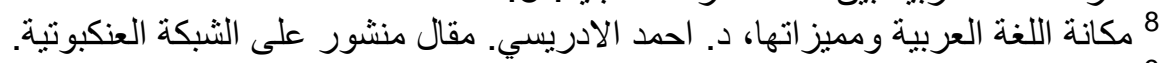

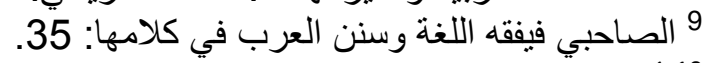

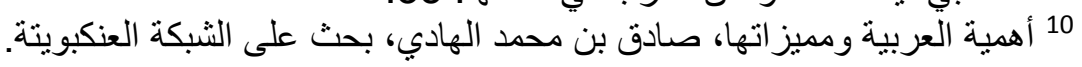




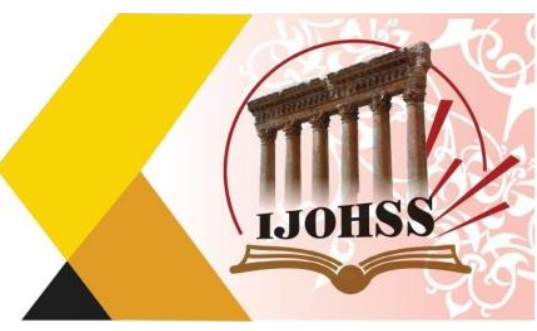

ولحن العامة، وصرنا أمام تراث جم في التصويب اللغوي على أساس المسار النحوي، والصرفي، والصائ والصوتي،

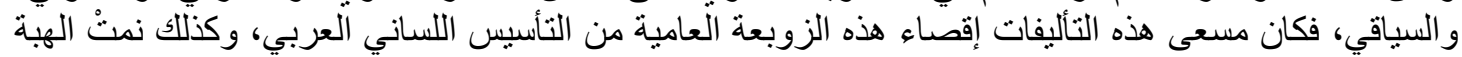

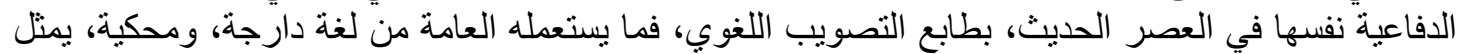

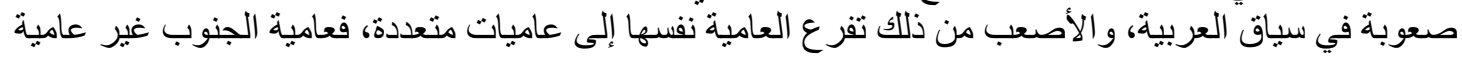

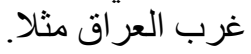

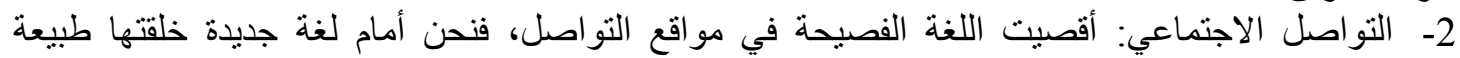

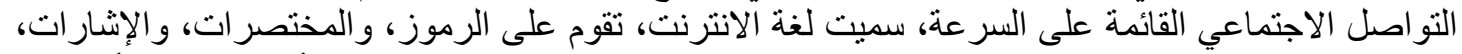

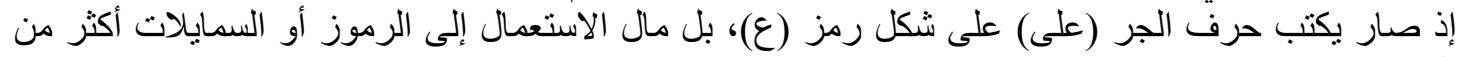

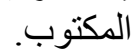

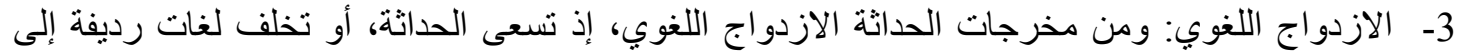

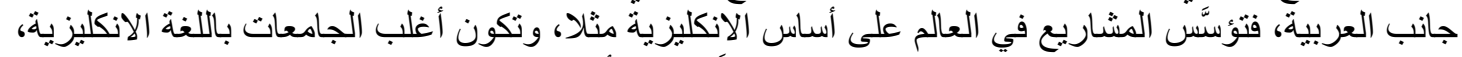

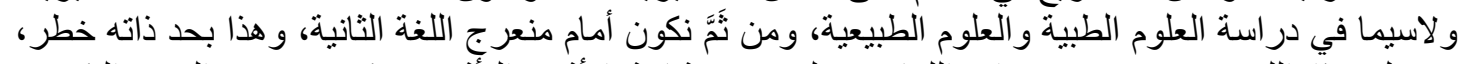

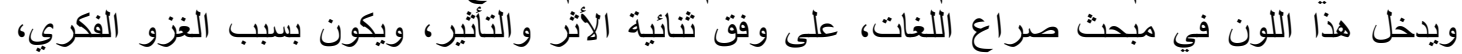

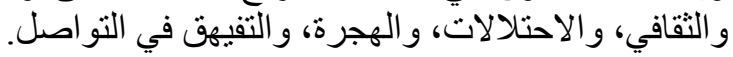

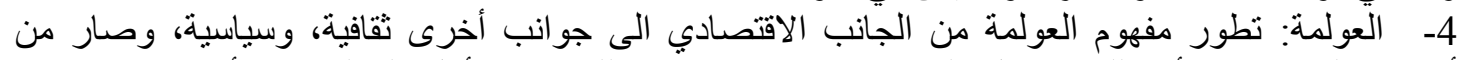

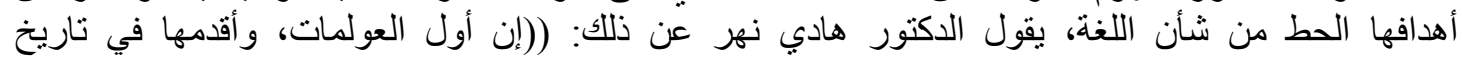

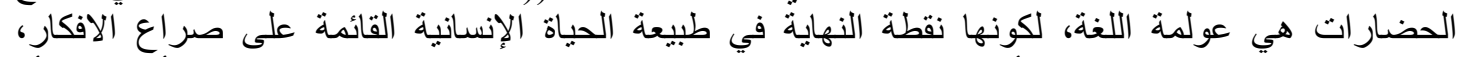

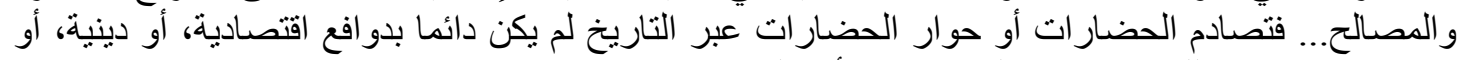

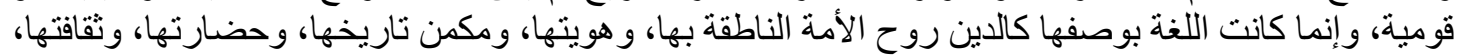

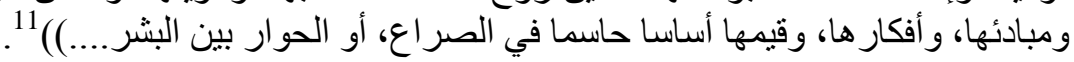

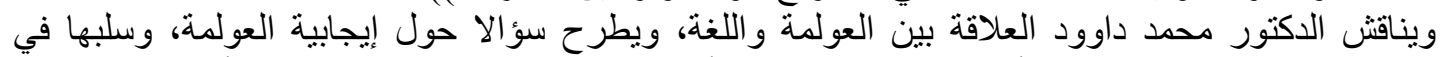

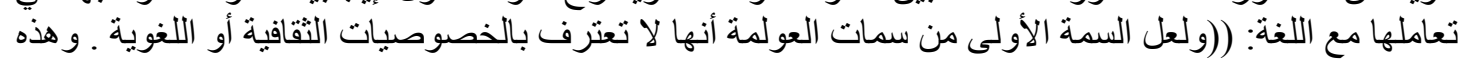

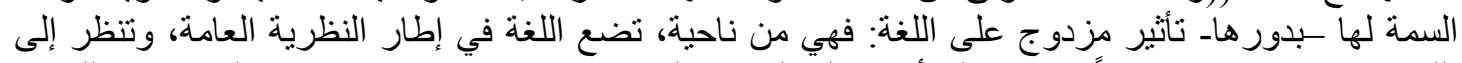

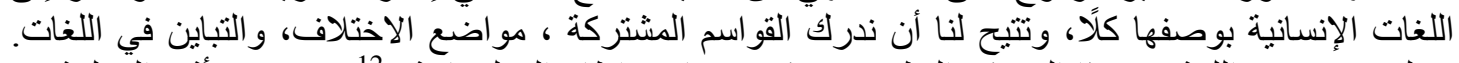

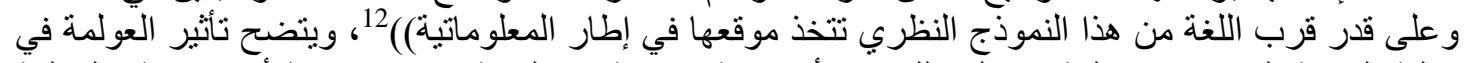

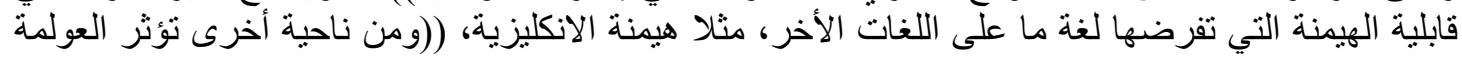

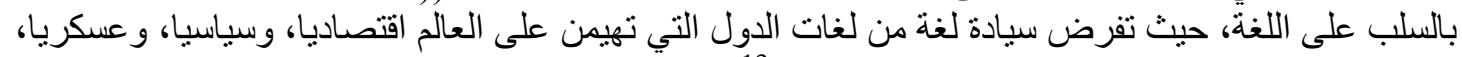

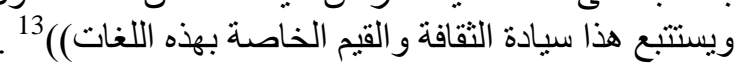

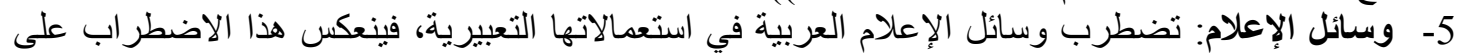

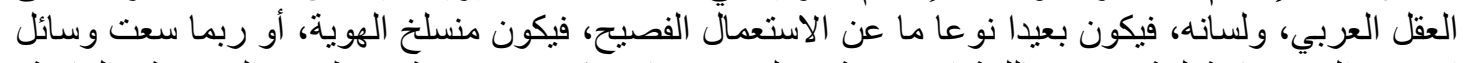

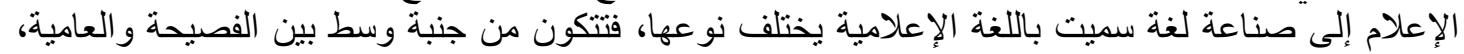

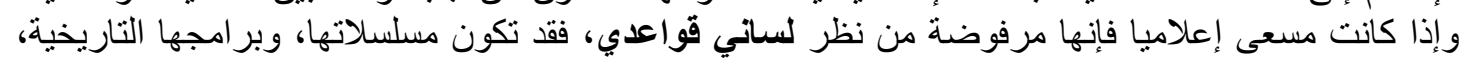

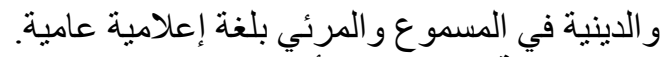

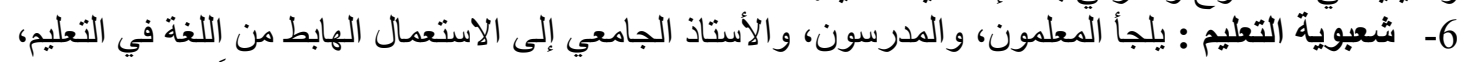

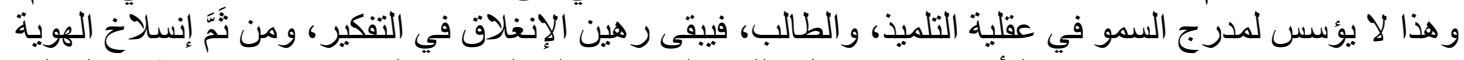

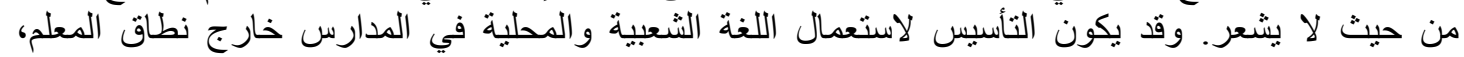

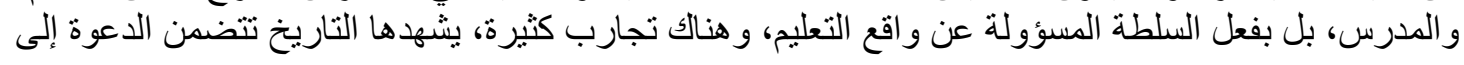

11 اللغة العربية في عالم متغير، دكتور هادي نهر، بحث منشور في وقائع المؤتمر العلمي الأول( اللغة العربية

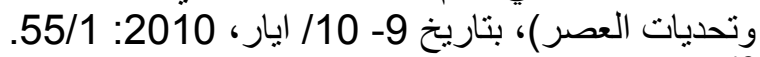
12 العربية و علم اللغة الحديث، دئ، دكتور محمد محمد داوود: 270. 13 العربية وعلم اللغة الحديث: 280. 


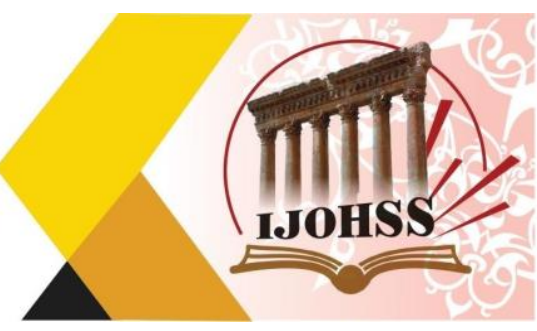

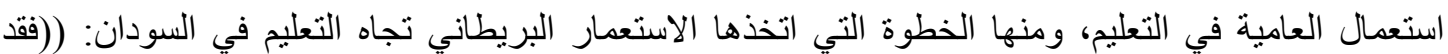

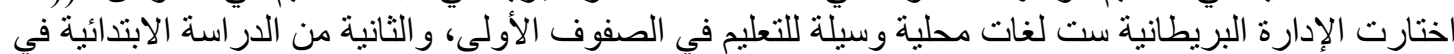

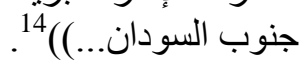

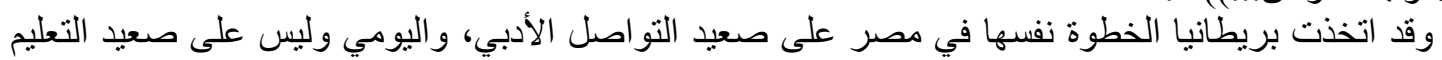

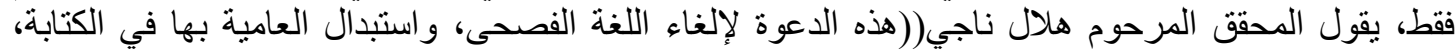

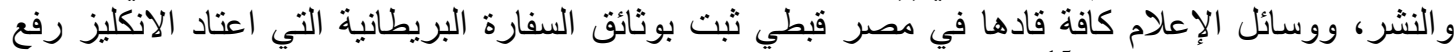

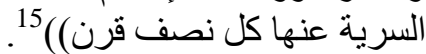

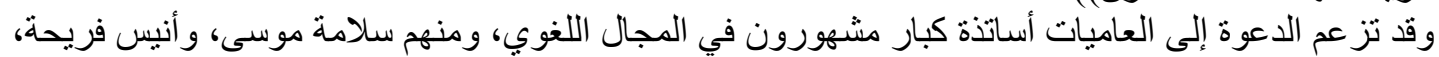

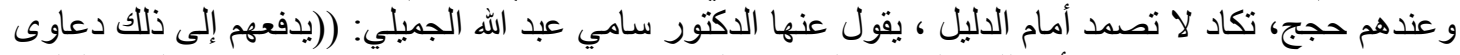

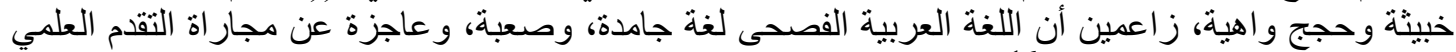

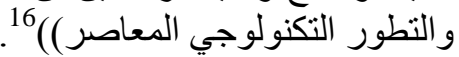

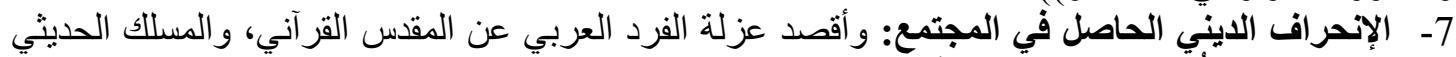

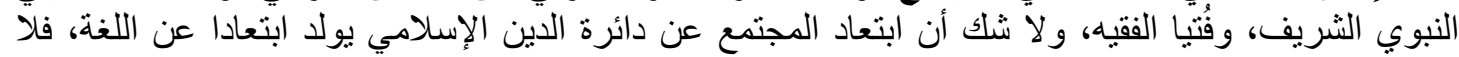

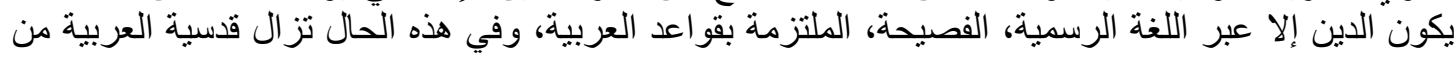

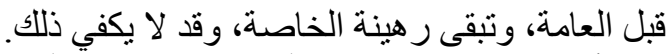

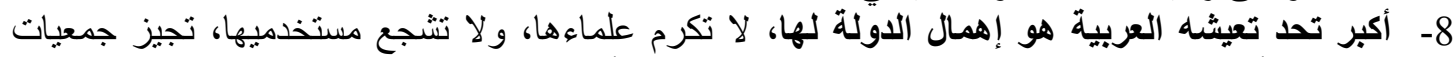

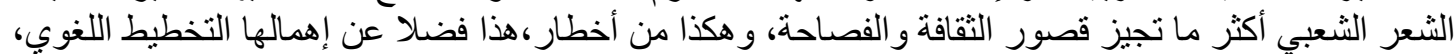

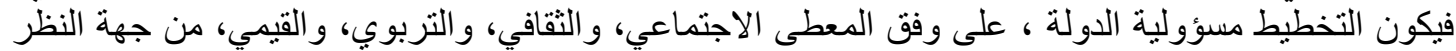

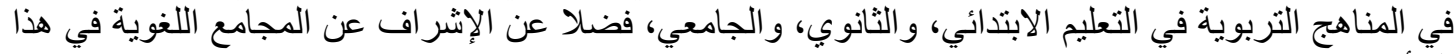

القسم الثالث : أسرار صمود العربية وخلودها

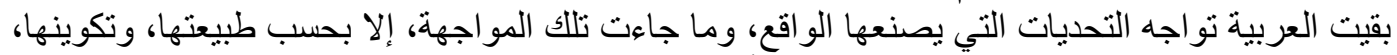

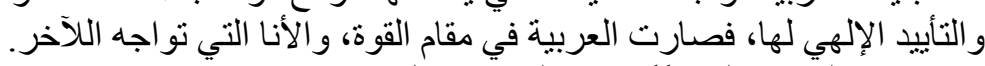

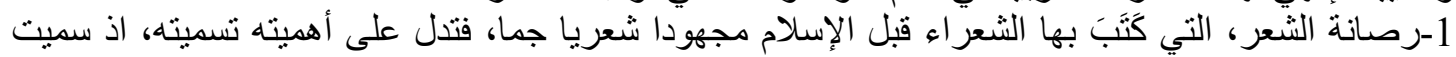

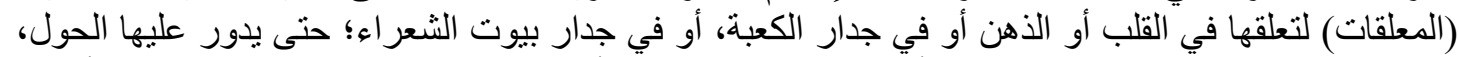

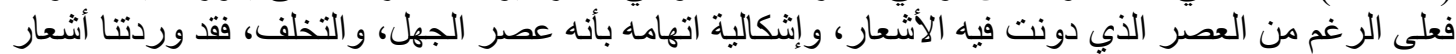

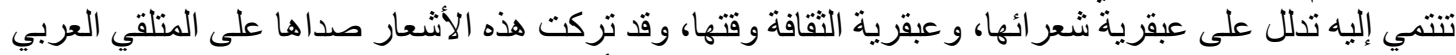

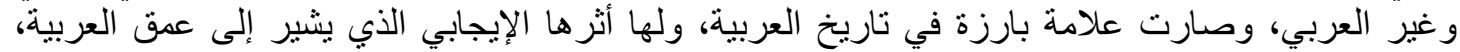

و وعبقها.

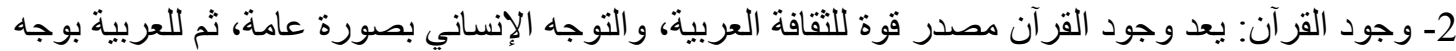

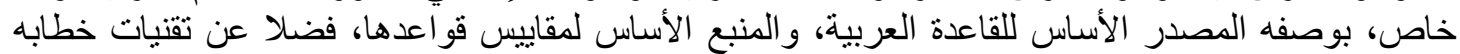

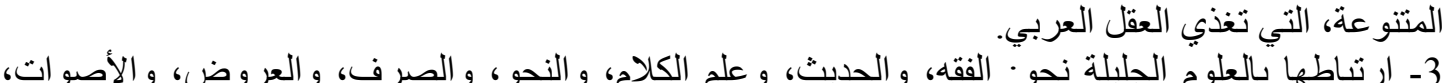

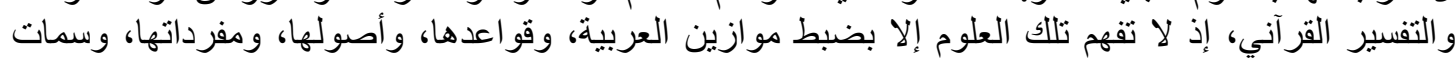

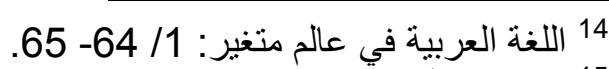

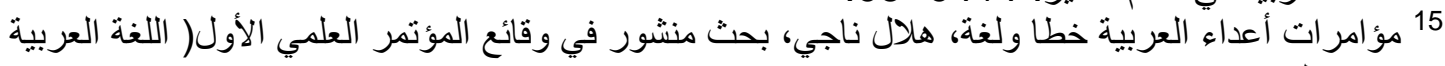

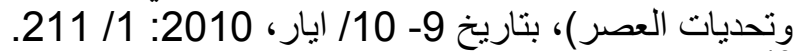

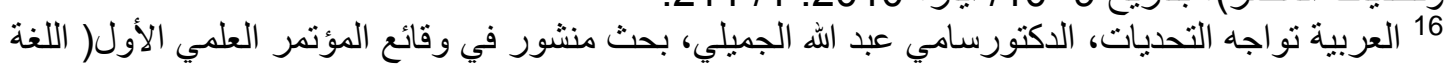

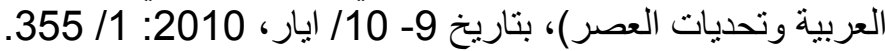




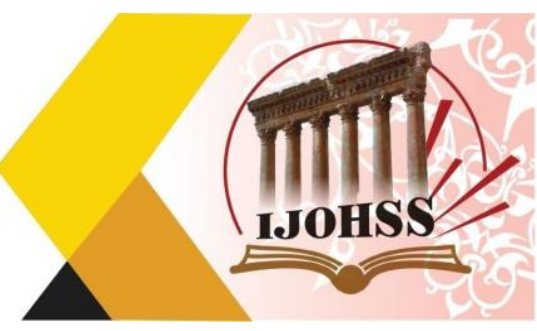

الفصاحة منها، و هذه الممارسة السابقة على العلوم، ومباحثها تجري في الدول العربية وغير العربية، مما يعزز من شأن العربية، ويسهم في بقائها.

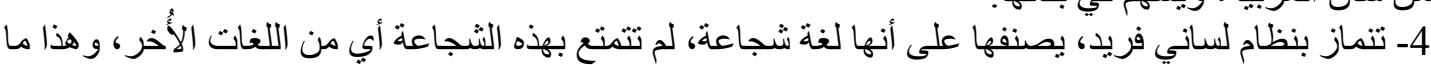

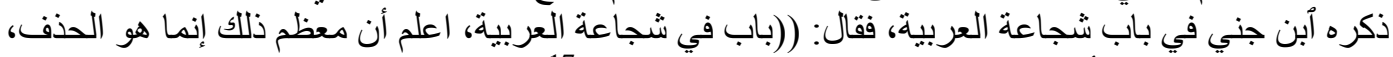

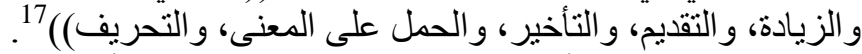

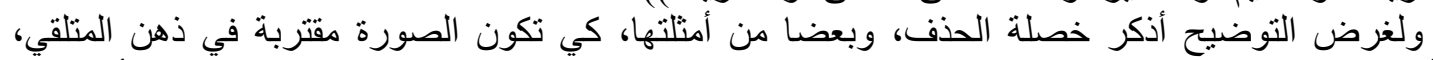

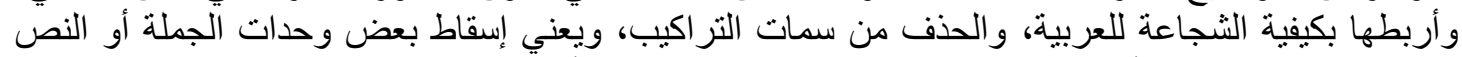

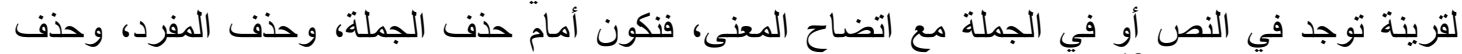

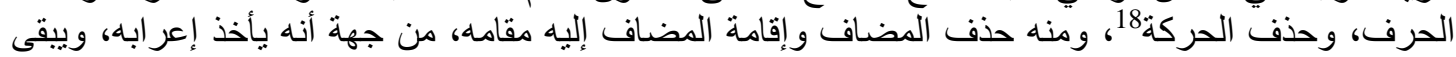

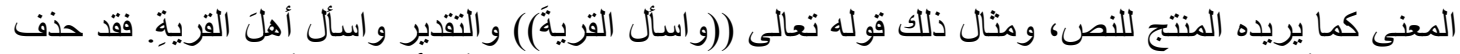

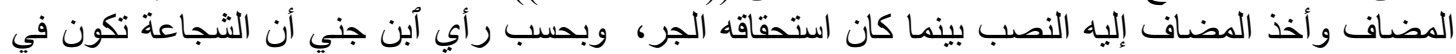

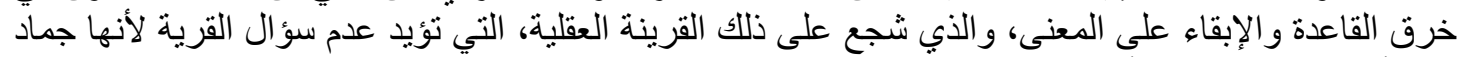

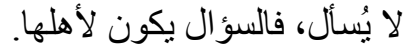

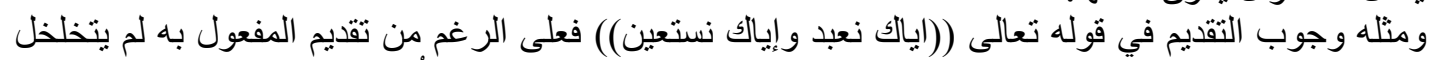

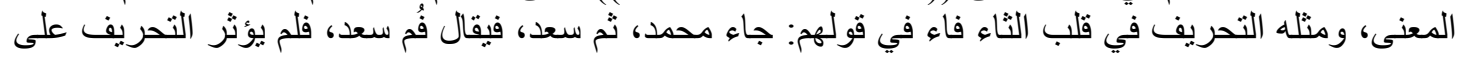
5- وجود مقاييس لغوية عربية، ومعنى المقاييس هنا، أنها حينما تم تأصبلها، وتسجيل علومها، وتقعيد قو اعدها

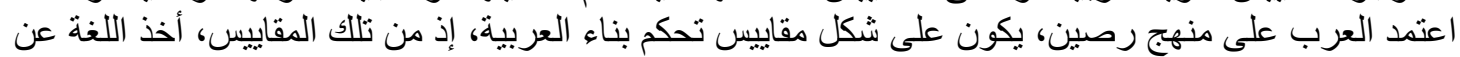

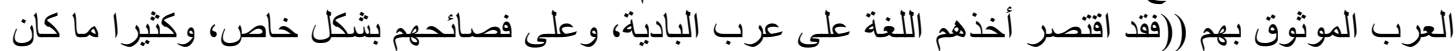

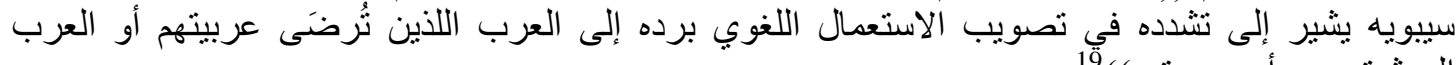

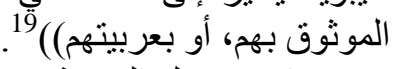

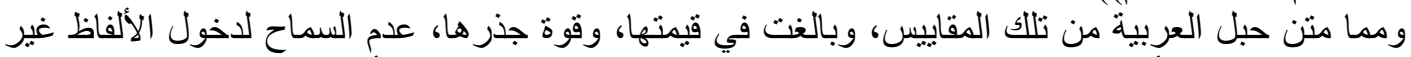

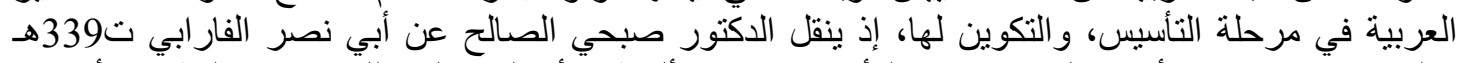

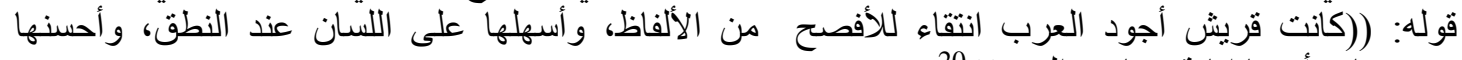

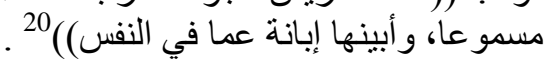

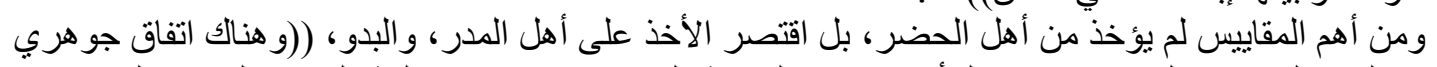

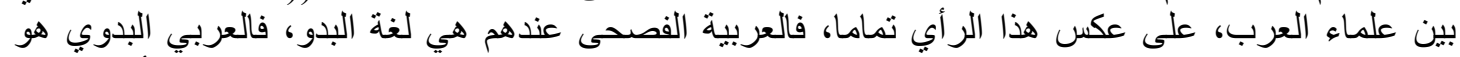

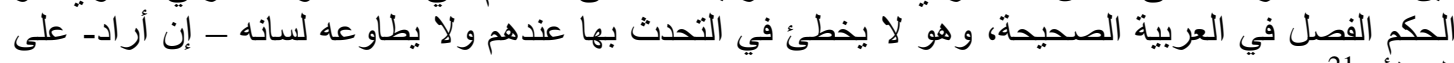

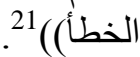

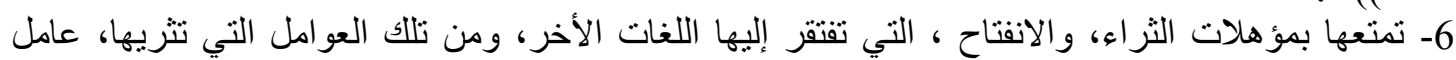

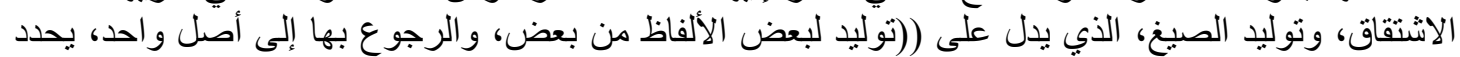

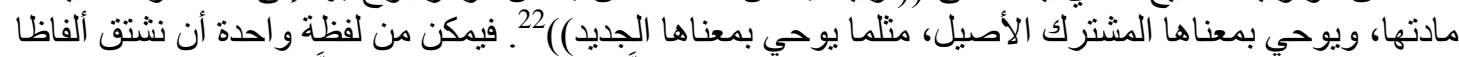

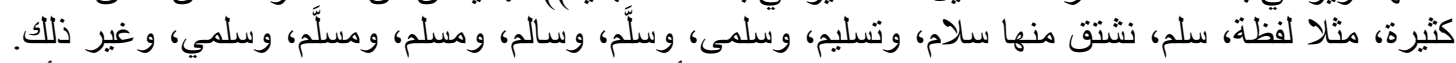

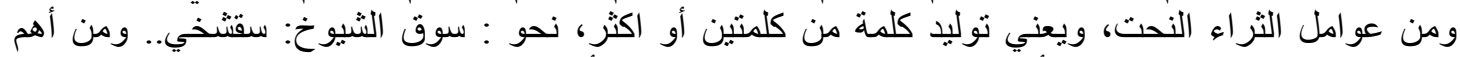

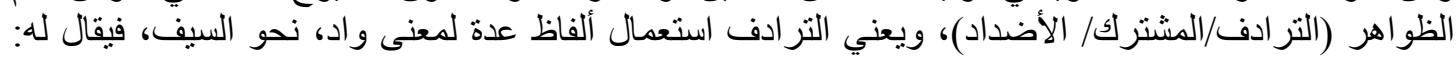

$$
\begin{aligned}
& \text { 17 - 17 الخصائص: } 140 \\
& 18 \text { ينظر: الخصائص: } 17 \text { الخص: 14/ 140 140. } \\
& 19 \text { در استات في فقه اللغة، الدكتور صبح صبحي الصالح: } 110 \\
& 20 \text { در اسات في فقه اللغة: } 112 \text { دراته اللغة الاكتور في }
\end{aligned}
$$

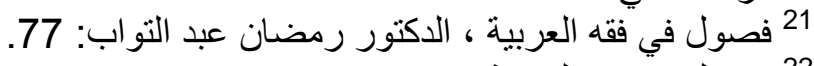

$$
\begin{aligned}
& 22 \text { فصول في فقه العربية: } 290 .
\end{aligned}
$$




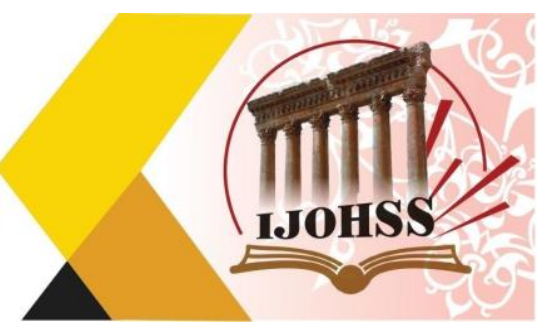

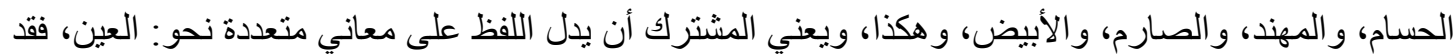

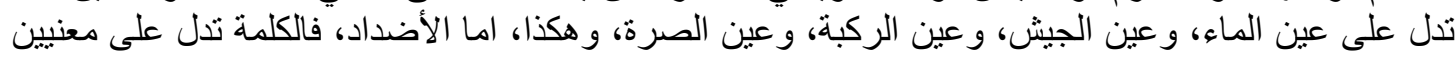

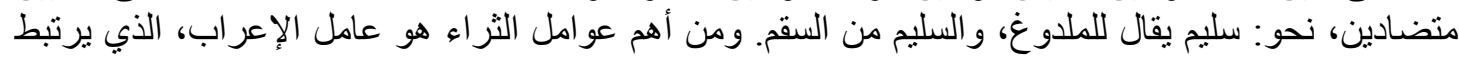

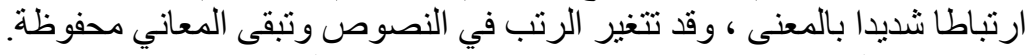

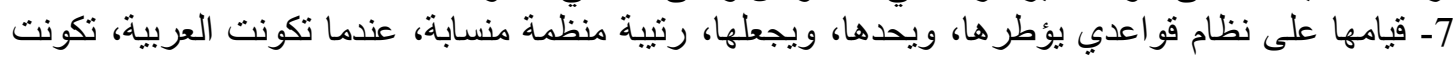

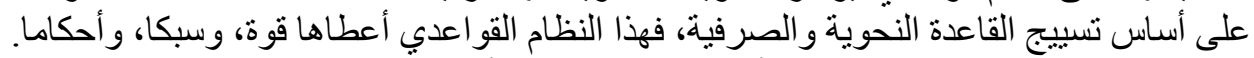

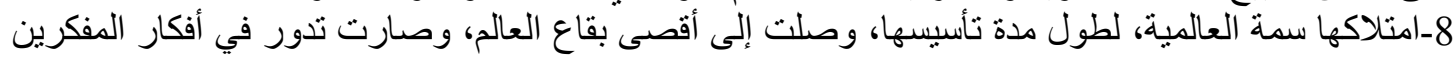

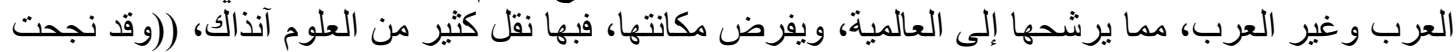

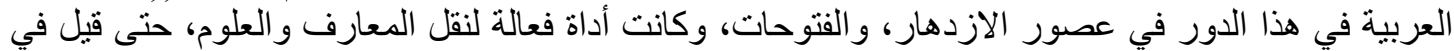

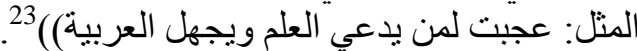

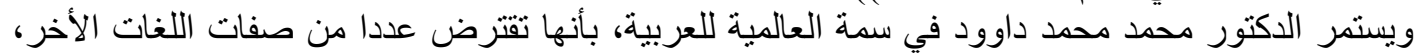

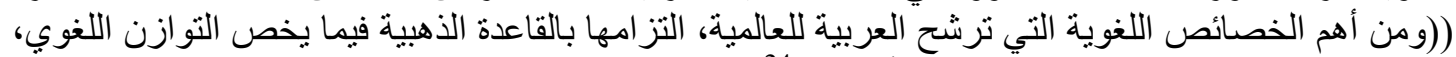

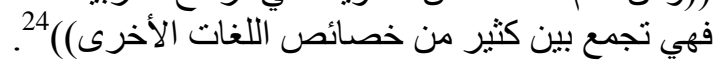

الخاتمة علاقة اللغة بالهوية....

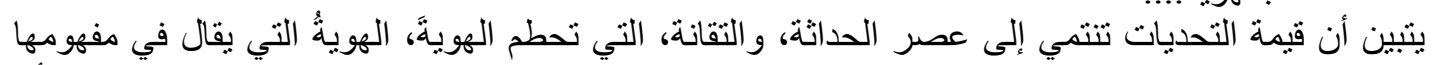

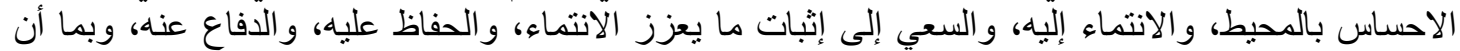

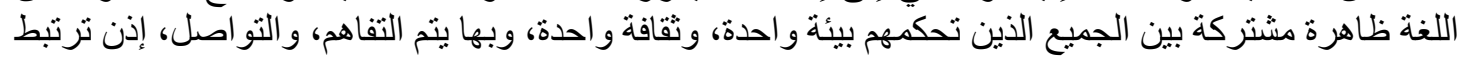

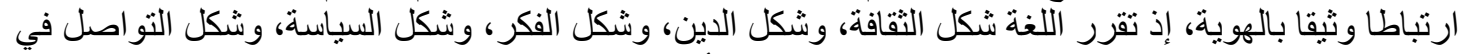

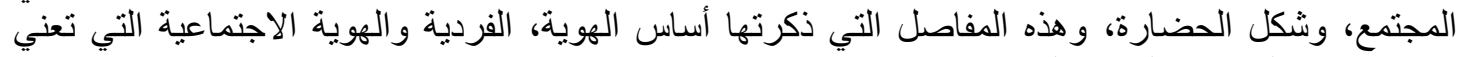
طريقة تفكير الجمهور الكثيرة الموحدة. فكلما حدث شر خ في طبيعة الجيعة اللغة، وشكلها، وبنياتها، وتكوناتها، وجزئياتها، ينعكس ذللك على الهوية الفردية،

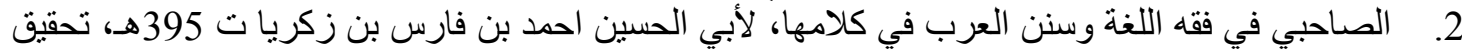

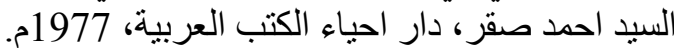
3. الخصائص، ابن جني ت 392هـ، تحقيق الدئن الدكتور عبد الحميد هنداوي، منشور ات محمد علي بيضون ، دار

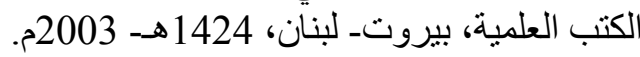
4. اكقه اللغة العربية ، مجد محمد الباكير، دار مجدلاوي للنشر والتوزيع، عمانـ الأردن للنشر والتوزيع، ط1،

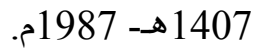
5

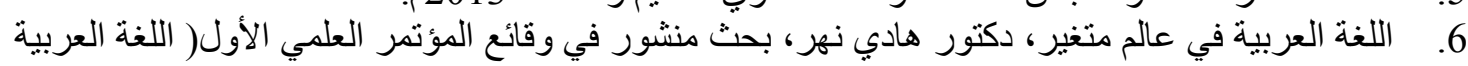

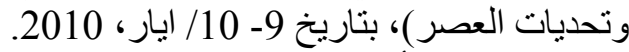

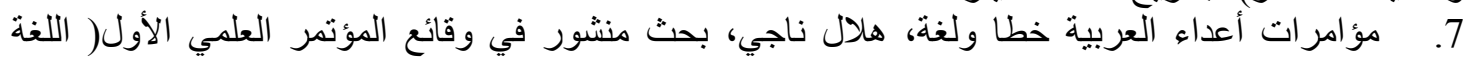

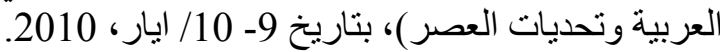
8. مكانة اللغة العربية ومميز اتها، د. احمد الادريسي. مقال منشور على الثبكة العنكبوتية.

$$
24 \text { العربية وعلم اللغة الحديث: } 281 \text { العزية } 281 .
$$




\section{المجلة الحولية اللعلوم الأنسانية والإمتماعية International Journal of Humanities and Social Sciences} website:www.ijohss.com Email:editor@ijohss.com ISSN: $2415-4822$
مارس 2021

Volume (19) March 2021

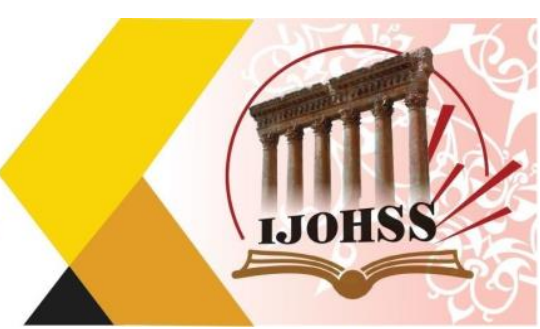

\section{References}

1. The importance of Arabic and its advantages, Sadiq bin Muhammad al-Hadi, research on the web.

2. Al-Sahbi on the jurisprudence of language and Sunan of the Arabs in her speech, by Abu Al-Hussein Ahmad bin Faris bin Zakaria 395 AH, verified by Mr. Ahmad Saqr, House of Revival of Arab Books, 1977 AD.

3. Characteristics, Ibn Jinni d. 392 AH, verified by Dr. Abd al-Hamid Hindawi, publications of Muhammad Ali Baydoun, Dar al-Kutub al-Ilmiyya, Beirut - Lebanon, 1424 AH - 2003 AD.

4. The Jurisprudence of the Arabic Language, Majd Muhammad al-Bakir, Majdalawi House for Publishing and Distribution, Amman - Jordan for Publishing and Distribution, 1st Edition, 1407 AH - 1987 AD.

5. The Poetic Language, Mahmoud Abbas Al-Akkad, Hindawi Foundation for Education and Culture, 2013 AD.

6. The Arabic Language in a Changing World, Dr. Hady Nahr, published research in the Proceedings of the First Scientific Conference (The Arabic Language and the Challenges of the Age), dated 9-10 / May, 2010.

7. Plots of the Enemies of Arabia, Line and Language, Hilal Naji, a research published in the Proceedings of the First Scientific Conference (The Arabic Language and the Challenges of the Age), dated 9-10 / May, 2010.

8. The status of the Arabic language and its advantages, d. Ahmed Al-Idrisi. Article published on the Internet.

9. The status of the Arabic language between languages, a contrasting study, Dr. Abdul-Majid Al-Tayeb Omar, Center for Scientific Research and Heritage Revival, Kingdom of Saudi Arabia, 2nd Edition, 10.11. 1437 AH.

12. Arabia faces challenges, Dr. Sami Abdullah Al-Jumaili, a research published in the Proceedings of the First Scientific Conference (The Arabic Language and the Challenges of the Age), on May 9-10, 2010. 


\section{المجلة اللحولية اللملوم الآنسانية والإمتماعية}

International Journal of Humanities and Social Sciences

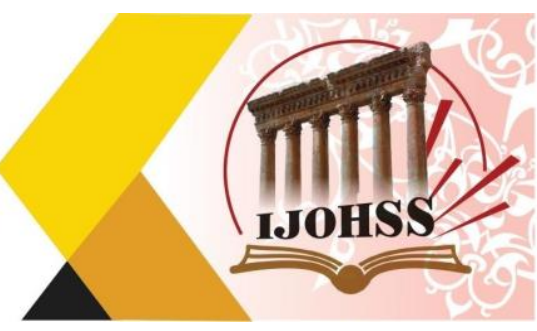

13. Arabic and Modern Linguistics, Dr. Muhammad Muhammad Daoud, Gharib House for Printing, Publishing and Distribution, Cairo, 2001.

14. Studies in Philology, Dr. Subhi Al-Saleh, House of Knowledge for the Millions, Beirut - Lebanon, 7th Edition, 1379 AH - 1960 AD.

15. Chapters on Arabic Jurisprudence, Dr. Ramadan Abdel Tawab, Al-Khanji Library in Cairo, 7th Edition, $1430 \mathrm{AH}-2009$ AD. 